\title{
Association between cytokine gene polymorphisms and tuberculosis in a Chinese population in Shanghai: a case-control study
}

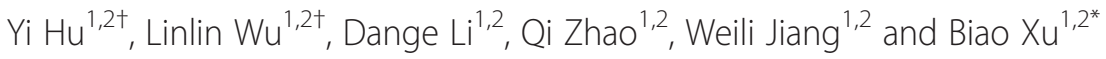

\begin{abstract}
Background: Polymorphisms in cytokine genes are known to influence cytokine levels, which may influence susceptibility to tuberculosis (TB) infection and disease. Differences in cytokine expression probably determine whether TB progresses, resolves, or becomes latent. In particular, the balance between the Th1 and Th2 cytokine responses influences the expression of disease in individuals with pulmonary TB (PTB). We performed a case-control study of 120 patients diagnosed with PTB, 240 with latent TB infection (LTBI), and 480 healthy controls (HC), to explore the association between polymorphisms in cytokine genes and a predisposition to Mycobacterium tuberculosis infection and TB disease.

Results: A single-gene analysis showed a dominant association between the AA genotype or A allele at nucleotide -874 of the interferon $\gamma$ (IFN- $\gamma$ ) gene and LTBI. The A allele at nucleotide -1082 of the interleukin 10 (IL-10) gene was significantly more common in PTB patients than in LTBI subjects. Moreover, the polymorphisms at IFN- $\gamma-874$ and IL10 -1082 were associated with protein levels of IFN- $\gamma$ and IL-10, respectively, in the PTB group. The genotype frequencies of other polymorphisms did not differ between the PTB patients, LTBI and HC subjects. Furthermore, combinations of polymorphisms with IFN- $\gamma-874$ were associated with LTBI, whereas combinations with $I L 10-1082$ were more likely associated with PTB.
\end{abstract}

Conclusions: There are positive associations between the IFN- $\gamma-874$ polymorphism and TB and between the IL10 - 1082 polymorphism and LTBI. Our data provide genetic evidence of the multiple disease hypothesis that many cytokine genes are involved in TB susceptibility.

Keywords: Tuberculosis, Latent tuberculosis infection, Cytokine, Polymorphism, Association

\section{Background}

Tuberculosis (TB) remains to be a leading cause of morbidity and mortality in developing countries, and the incidence of the disease is increasing in developed countries [1]. One third of the world's population is infected by Mycobacterium tuberculosis (Mtb), but only about $5 \%$ of infected individuals develop the disease within the first year of infection and another 5\% develop the disease later in life. Candidate gene and association studies have identified various host genetic factors that

\footnotetext{
* Correspondence: bxu@shmu.edu.cn

${ }^{\dagger}$ Equal contributors

'Department of Epidemiology, School of Public Health, Fudan University, 138 Yi Xue Yuan Road, Shanghai 200032, China

${ }^{2}$ Key Laboratory of Public Health Safty (Fudan University), Ministry of

Education, Shanghai, China
} ing the host genes responsible for susceptibility and resistance to TB may lead towards better understanding of the pathogenesis of TB and development of prophylactic or treatment strategies.

The cytokines produced at the site of disease after the interaction between $\mathrm{T}$ lymphocytes and infected macrophages are essential for the pathogenesis of TB [4]. The course of Mtb infection is regulated by two distinct T-cell cytokine patterns. T-helper 1 (Th1) cytokines, interleukin (IL)-2 and interferon $\gamma$ (IFN- $\gamma$ ), are associated with resistance to infection, whereas T-helper 2 (Th2) cytokines, $I L-4$ and $I L-13$, are associated with progressive disease [5]. Polymorphisms in several cytokine genes have been described and shown to influence gene transcription, leading to inter individual variations in cytokine

play significant roles in susceptibility to TB [2,3]. Identify- 
production [6]. Cytokine gene polymorphisms have been shown to be involved in the susceptibility, the severity and clinical outcomes of several diseases, including infectious diseases [7]. A single nucleotide polymorphism (SNP) located in the first intron of human the IFN- $\gamma$ gene $(+874 \mathrm{~T} / \mathrm{A})$ putatively influences the secretion of the cytokine, with an impact on the outcomes of infections. This polymorphism displays variable associations with TB disease susceptibility and severity [8] in some ethnic populations but not in others [9]. A specific nucleotide polymorphism within the promoter region of $I L-10$ (1082A/G) is also highly variable. This polymorphism alters the level of this cytokine and possibly the Th1/Th2 balance, with major implications for TB infection [6]. Data on the association between the 1082G/A SNP of $I L-10$ and TB are also highly variable. Among the proinflammatory cytokines, tumor necrosis factor $\alpha(T N F-\alpha)$ SNP -308 is associated with either TB susceptibility or severity [10]. Furthermore, because the immune interactions that determine TB disease susceptibility and severity are complex, multigene combinations may give more meaningful information than single SNPs in determining disease outcomes [11].

Most studies have not differentiated TB disease according to its natural history. Therefore, it is difficult to evaluate the differences in their results. Our study groups were stratified into latent TB infection (LTBI) and active TB disease. We investigated the influence of IFN- $\gamma, T N F-\alpha$, and interleukin cytokine gene polymorphisms on the susceptibility of individuals to LTBI and TB disease in terms of the cytokine levels expressed and their associations with ex vivo stimulate cytokine and pulmonary TB in a population from Shanghai. We also analyzed combinations of cytokine gene polymorphisms.

\section{Results}

\section{Demographic characteristics of the study groups}

The study subject groups consisted of 120 newly diagnosed PTB patients, 240 subjects with LTBI, and 480 healthy control (HC) subjects. The mean ages and sex distributions of the patients and controls did not differ. The Bacille Calmette- Guérin (BCG) scar was observed $87.5 \%$ of PTB patients, $93.8 \%$ of the LTBI group, and 94.0\% of $\mathrm{HC}$ subjects (Table 1 ).

\section{Associations between Th1 cytokine polymorphisms and TB susceptibility}

The genotype distributions of the IFN- $\gamma-874 \mathrm{~A} / \mathrm{T}$ SNP and CA repeat did not deviate from Hardy-Weinberg equilibrium in any subjects. At the IFN- $\gamma$ T874A polymorphic site, the frequency of the A allele was 1.30 -fold higher in the LTBI than in the $\mathrm{HC}$ subjects $(\mathrm{OR}=1.30$; 95\% CI: 1.020-1.664). The LTBI group had a significantly higher proportion of the AA genotype than the healthy group $(\mathrm{OR}=1.39$; 95\% CI: $1.011-1.930)$. However, the PTB and LTBI groups had very similar frequency distributions for the other $-874 \mathrm{~A} / \mathrm{T}$ genotypes and alleles. We identified 10 alleles for the CA repeat polymorphism (ranging from 10 to $19 \mathrm{CA}$ repeats). The 12,13 , and $15 \mathrm{CA}$ repeats were found to be the most common alleles in the study groups. The three groups displayed similar distributions of the CA repeats, although the TB group contained a significantly higher proportion of the 12-repeat allele in the LTBI group $(\mathrm{OR}=1.71 ; 95 \% \mathrm{CI}: 1.074-2.728)$. A haplotype analysis revealed no significant differences in the IFN- $\gamma$ A-12 haplotype frequency between the three groups. The in vitro production of IFN- $\gamma$ correlated with the IFN- $\gamma-874$ polymorphism. IFN- $\gamma$ levels were significantly lower in the LTBI and PTB groups than in the HC group ( $p=0.001$; Table 2, Figure 1 ).

In $T N F-\alpha$, the frequencies of the TNF- $\alpha+488 \mathrm{~A}$, $-238 \mathrm{~A}$, and $-308 \mathrm{~A}$ alleles were $6.5 \%, 3.3 \%$, and $4.7 \%$ in the $\mathrm{HC}$ group, $7.1 \%, 3.3 \%$, and $5.2 \%$ in the LTBI group, respectively, and $10.0 \%, 4.6 \%$, and $10.8 \%$ in the PTB group, respectively. The number of individuals homozygous for $+488 \mathrm{~A}$ was low and there were no homozygotes for $-238 \mathrm{~A}$ or $-308 \mathrm{~A}$ in the PTB group. No significant differences in the frequencies of the three polymorphic variants of $T N F-\alpha$ were observed between the healthy control and LTBI groups or between the LTBI and PTB groups. The haplotype frequencies were similar between the three groups. The levels of TNF- $\alpha$ were not related to the TNF- $\alpha$ polymorphisms and were similar between the three groups (Table 2, Figure 1).

At the -330 SNP of $I L-2$, a significant negative association was evident between the TT genotype and the PTB group compared with the LTBI group (28.3\% vs $44.6 \%$, $\mathrm{OR}=0.49,95 \% \mathrm{CI}: 0.297-0.806$ ), whereas the G allele was significantly overrepresented in the PTB group. A

Table 1 Baseline information on the study groups

\begin{tabular}{llllll}
\hline & HC $(\mathbf{n}=\mathbf{4 8 0})$ & LTBI $(\mathbf{n}=\mathbf{2 4 0})$ & PTB $(\mathbf{n}=\mathbf{1 2 0})$ & $\mathbf{X}^{\mathbf{2}} \mathbf{F}$ & $\mathbf{p}$ value \\
\hline Age $($ mean \pm SD) & $41.0 \pm 19.9$ & $43.0 \pm 18.1$ & $46.0 \pm 12.2$ & 0.141 & 0.234 \\
Sex (male) N(\%) & $294(61.2)$ & $147(61.2)$ & $74(61.7)$ & 0.008 & 0.996 \\
BCG scar N(\%) & $451(94.0)$ & $225(93.8)$ & $105(87.5)$ & 6.440 & $0.040^{*}$ \\
\hline
\end{tabular}

Notes: SD, standard deviation; HC, healthy controls; LTBI, subjects with latent tuberculosis infection; PTB, patients with pulmonary tuberculosis; BCG, Bacillus Calmette-Guérin vaccine.

${ }^{*} \mathrm{p}<0.05$. 
Table 2 Allele, genotype, and haplotype of Th1, Th2, and Th17 cytokine polymorphisms signficantly associated with LTBI and TB

\begin{tabular}{|c|c|c|c|c|c|c|c|c|}
\hline & & & LTBI & PTB & LTBI.VS.HCS & & PTB.vs.LTBI & \\
\hline & & $N(\%)$ & $N(\%)$ & $N(\%)$ & $\mathrm{OR}(95 \% \mathrm{Cl})$ & $p$ value & OR(95\%Cl) & $p$ value \\
\hline IFN-r & Genotypes & & & & & & & \\
\hline$+874(\mathrm{~A} / \mathrm{T})$ & AA & $212(44.2)$ & $126(52.5)$ & $74(61.7)$ & 1.39(1.011-1.930) & $0.035^{*}$ & $1.46(0.910-2.337)$ & 0.099 \\
\hline & AT & 201(41.9) & $88(36.7)$ & $36(30.0)$ & $0.80(0.576-1.119)$ & 0.131 & $0.74(0.448-1.213)$ & 0.210 \\
\hline & $\pi$ & $67(14.0)$ & $26(10.8)$ & $10(8.3)$ & $0.75(0.443-1.235)$ & 0.171 & $0.75(0.311-1.676)$ & 0.456 \\
\hline & Alleles & & & & & & & \\
\hline & A & $625(65.1)$ & $340(70.8)$ & 184(76.7) & $1.30(1.020-1.664)$ & $0.029^{*}$ & $1.35(0.934-1.974)$ & 0.097 \\
\hline & $\mathrm{T}$ & $335(34.9)$ & $140(29.2)$ & $56(23.3)$ & $0.77(0.601-0.980)$ & $0.029^{*}$ & $0.74(0.507-1.071)$ & 0.097 \\
\hline CA repeats & Alleles & & & & & & & \\
\hline & 12 & $641(66.8)$ & $340(70.8)$ & 186(77.5) & $1.21(0.946-1.547)$ & 0.119 & $1.71(1.074-2.728)$ & $0.017^{*}$ \\
\hline & Non-12 & 319(33.2) & $140(29.2)$ & $54(22.5)$ & $0.83(0.647-1.057)$ & 0.119 & $0.58(0.367-0.931)$ & $0.017^{*}$ \\
\hline Haplotypes & & & & & & & & \\
\hline (+874 and CA repeats) & $A-12$ & 167(17.4) & $103(21.5)$ & $60(25.0)$ & $1.30(0.975-1.721)$ & 0.063 & $1.22(0.847-1.757)$ & 0.284 \\
\hline & $\mathrm{T}-12$ & 174(18.1) & 75(15.6) & $30(12.5)$ & $0.84(0.613-1.134)$ & 0.237 & $0.77(0.472-1.238)$ & 0.263 \\
\hline & A-non12 & $324(33.8)$ & $175(36.5)$ & $98(40.8)$ & $1.13(0.889-1.425)$ & 0.309 & $1.20(0.864-1.672)$ & 0.254 \\
\hline & T-non12 & 295(30.7) & $127(26.5)$ & $52(21.7)$ & $0.81(0.629-1.043)$ & 0.093 & $0.77(0.521-1.126)$ & 0.161 \\
\hline TNF-a & Haplotypes & & & & & & & \\
\hline$(238,308,488)$ & $A-A-A$ & $1(0.2)$ & $1(0.4)$ & $0(0)$ & $1.996(0.124-32.047)$ & 1.000 & - & - \\
\hline & $A-A-G$ & $205(42.7)$ & $102(42.5)$ & $0(0)$ & $0.984(0.720-1.346)$ & 0.922 & $0.54(0.477-0.599)$ & $0.000^{*}$ \\
\hline & $A-G-A$ & $0(0)$ & $0(0)$ & $43(35.8)$ & - & & $4.12(3.390-5.000)$ & $0.000^{*}$ \\
\hline & $A-G-G$ & $20(4.2)$ & $10(4.2)$ & $12(10.0)$ & $0.996(0.459-2.162)$ & 0.880 & $2.56(1.071-6.099)$ & $0.029^{*}$ \\
\hline & G-A-A & $1(0.2)$ & $1(0.4)$ & $0(0)$ & $1.996(0.124-32.047)$ & 1.000 & - & \\
\hline & $G-A-G$ & $13(2.7)$ & $7(2.9)$ & $53(44.2)$ & $1.075(0.423-2.730)$ & 0.880 & $26.33(11.439-60.61)$ & $0.000^{*}$ \\
\hline & G-G-A & $210(43.8)$ & 105(43.8) & $5(4.2)$ & $0.993(0.727-1.356)$ & 0.963 & $0.06(0.022-0.142)$ & $0.000^{*}$ \\
\hline & $\mathrm{G}-\mathrm{G}-\mathrm{G}$ & $30(6.3)$ & $15(6.3)$ & $7(5.8)$ & 0.996(0.525-1.888) & 0.989 & $0.93(0.368-2.344)$ & 0.876 \\
\hline IL2 & Genotype & & & & & & & \\
\hline$-330(\mathrm{~T} / \mathrm{G})$ & GG & $44(9.2)$ & $29(12.1)$ & $30(25.0)$ & $1.210(0,742-1.969)$ & 0.445 & $2.43(1.376-4.275)$ & $0.002^{*}$ \\
\hline & GT & 256(53.3) & 104(43.3) & $56(46.7)$ & $0.669(0.489-0.914)$ & $0.011^{*}$ & $1.14(0.737-1.777)$ & 0.549 \\
\hline & $\pi$ & $180(37.5)$ & 107(44.6) & $34(28.3)$ & $1.34(0.966-1.858)$ & 0.067 & $0.49(0.297-0.806)^{*}$ & $0.003^{*}$ \\
\hline & Alleles & & & & & & & \\
\hline & G & $344(35.8)$ & 162(33.8) & $116(48.3)$ & $0.91(0.724-1.149)$ & 0.435 & 1.84(1.339-2.519) & $0.000^{*}$ \\
\hline & T & $616(64.2)$ & $318(66.2)$ & 124(51.7) & $1.10(0.865-1.391)$ & 0.435 & $0.545(0.392-0.757)^{*}$ & $0.000^{*}$ \\
\hline-330 and -160 & Haplotypes & & & & & & & \\
\hline & G-G & $205(21.4)$ & $97(20.3)$ & $48(20.1)$ & $0.93(0.703-1.232)$ & 0.615 & $0.99(0.655-1.475)$ & 0.948 \\
\hline & G-T & $99(10.3)$ & $68(14.1)$ & $32(13.5)$ & $1.44(1.015-2.021)$ & $0.031^{*}$ & $0.93(0.573-1.493)$ & 0.761 \\
\hline & $\mathrm{T}-\mathrm{G}$ & $339(41.6)$ & 95(39.5) & $95(39.4)$ & $0.45(0.344-0.590)$ & $0.000^{*}$ & $2.66(1.856-3.793)$ & $0.000^{*}$ \\
\hline & $\mathrm{T}-\mathrm{T}$ & $317(26.7)$ & $220(26.2)$ & $65(27)$ & $1.72(1.362-2.161)$ & $0.000^{*}$ & $0.44(0.308-0.622)$ & $0.000^{*}$ \\
\hline IL-12 & genotypes & & & & & & & \\
\hline$-1188(\mathrm{~A} / \mathrm{C})$ & AA & $270(56.2)$ & $162(67.5)$ & $80(66.7)$ & $1.62(1.154-2.269)$ & $0.004^{*}$ & $0.96(0.590-1.582)$ & 0.874 \\
\hline & $A C$ & 177(36.9) & $65(27.1)$ & $33(27.5)$ & $0.66(0.460-0.933)$ & $0.009^{*}$ & $1.02(0.603-1.712)$ & 0.933 \\
\hline & $\mathrm{CC}$ & $33(6.9)$ & $13(5.4)$ & $7(5.8)$ & $0.78(0.367-1.55)$ & 0.451 & $1.08(0.355-3.011)$ & 0.871 \\
\hline & Alleles & & & & & & & \\
\hline & A & $717(74.7)$ & 389(81.0) & 193(80.4) & $1.49(1.097-1.922)$ & $0.007^{*}$ & $0.96(0.639-1.456)$ & 0.841 \\
\hline
\end{tabular}


Table 2 Allele, genotype, and haplotype of Th1, Th2, and Th17 cytokine polymorphisms signficantly associated with LTBI and TB (Continued)

\begin{tabular}{|c|c|c|c|c|c|c|c|c|}
\hline & C & $243(25.3)$ & $91(19.0)$ & 47(19.6) & $0.69(0.520-0.911)$ & $0.007^{*}$ & $1.04(0.687-1.565)$ & 0.841 \\
\hline IL-10 & Genc & & & & & & & \\
\hline \multirow[t]{3}{*}{$-1082(\mathrm{~A} / \mathrm{G})$} & AA & $262(54.6)$ & $136(56.7)$ & $82(68.3)$ & $1.09(0.787-1.506)$ & 0.596 & $1.65(1.016-2.699)$ & $0.033^{*}$ \\
\hline & $A G$ & 196(40.8) & $86(35.8)$ & $34(28.3)$ & $0.81(0.579-1.128)$ & 0.195 & $0.71(0.425-1.168)$ & 0.155 \\
\hline & GG & $22(4.6)$ & $18(7.5)$ & $4(3.4)$ & $1.69(0.834-3.368)$ & 0.107 & $0.43(0.103-1.335)$ & 0.120 \\
\hline \multirow[t]{5}{*}{-1082 and -819} & Hapl & & & & & & & \\
\hline & $A-C$ & $205(21.4)$ & $116(24.2)$ & $58(24.2)$ & $1.17(0.896-1.533)$ & 0.235 & $0.24(0.683-1.456)$ & 1.000 \\
\hline & $A-T$ & $100(10.4)$ & 174(36.2) & $95(39.6)$ & $4.95(3.707-6.608)$ & $0.000^{*}$ & $1.15(0.826-1.604)$ & 0.383 \\
\hline & $\mathrm{G}-\mathrm{C}$ & $400(41.7)$ & $56(11.7)$ & 26(10.8) & $0.19(0.134-0.254)$ & $0.000^{*}$ & $0.91(0.533-1.525)$ & 0.740 \\
\hline & G-T & 255(26.6) & $134(27.9)$ & $61(25.4)$ & $1.07(0.830-1.378)$ & 0.601 & $0.88(0.607-1.268)$ & 0.477 \\
\hline IL-6 & Allel & & & & & & & \\
\hline \multirow[t]{6}{*}{$-174(G / C)$} & G & $674(70.2)$ & $363(75.6)$ & 182(75.8) & $1.32(1.017-1.707)$ & $0.031^{*}$ & $1.01(0.695-1.481)$ & 0.951 \\
\hline & $C$ & 286(29.8) & $117(24.4)$ & $58(24.2)$ & $0.76(0.586-0.982)$ & $0.031^{*}$ & $0.99(0.675-1.439)$ & 0.951 \\
\hline & gen & & & & & & & \\
\hline & GG & $247(51.5)$ & $131(54.6)$ & $66(55.0)$ & $1.03(0.749-1.429)$ & 0.429 & $1.02(0.640-1.620)$ & 0.940 \\
\hline & GC & $180(37.5)$ & $101(42.1)$ & $50(41.7)$ & $1.21(0.871-1.681)$ & 0.235 & $0.98(0.614-1.569)$ & 0.940 \\
\hline & CC & $53(11.0)$ & $8(3.3)$ & $4(3.3)$ & $0.36(0.144-0.793)$ & $0.000^{*}$ & $1.00(0.216-3.826)$ & 1.000 \\
\hline
\end{tabular}

Notes: HC, healthy controls; LTBI, subjects with latent tuberculosis infection; PTB, patients with pulmonary tuberculosis; INF- $\gamma$, interferon $\gamma$; TNF-a, tumor necrosis factor $\alpha$; IL-2, interleukin 1; IL-12, interleukin 12; IL-10, interleukin 10; IL-4, interleukin 4; IL-6, interleukin 6.

${ }^{*} p<0.05$.

trend towards a reduced IL-2 response was observed with the -330 GG genotype compared with the TG and TT genotypes in culture filtrate (CFA)-stimulated cultures of peripheral blood mononuclear cells (PBMCs) from $\mathrm{HC}$ subjects $(p=0.35)$. No significant differences in $I L-2$ levels were observed between the variant genotypes of the IL2 polymorphism in the $\mathrm{HC}$ or the LTBI groups (Figure 1).

\section{Associations between Th2 cytokine polymorphisms and TB susceptibility}

Homozygosity for the $I L-10$ - 1082A allele occurred at a higher frequency in the PTB group than in the LTBI group $(\mathrm{OR}=1.61,95 \% \mathrm{CI}: 1.071-2.438)$, indicating a significant association between $\mathrm{TB}$ and the $I L-10-1082$ AA genotype $(\mathrm{OR}=1.65,95 \% \mathrm{CI}$ : 1.061-2.699). No statistically significant difference was observed in the distributions of the $I L-10-1082$ genotypes in the LTBI and $\mathrm{HC}$ groups $(p>0.05)$. The distributions of the $I L-10-$ 819 genotypes were similar in the PTB patients, LTBI and $\mathrm{HC}$ subjects $(p>0.05)$. There was no linkage between the $-1082 \mathrm{~A} / \mathrm{G}$ and $-819 \mathrm{C} / \mathrm{T}$ IL10 polymorphisms. The frequency of the $I L-10-1082 \mathrm{G} /-819 \mathrm{C}$ haplotype was lower in the LTBI subjects than in the HC (11.6\% vs $41.6 \%$, respectively; $\mathrm{OR}=0.19,95 \% \mathrm{CI}: 0.134-0.254$ ), whereas the frequency of the $I L-10-1082 \mathrm{~A} /-819 \mathrm{~T}$ haplotype was higher $(36.2 \%$ vs $10.3 \%$, respectively; OR = 4.95, 95\% CI: 3.707-6.608). However, the haplotype distributions did not differ significantly between the LTBI and
PTB subjects and the $I L-10$ levels did not differ among the variant genotypes of the $I L-10$ gene polymorphisms in the two groups (Table 3 ).

The allele and genotype frequencies of the $I L-4$ variable number tandem repeat (VNTR) polymorphism did not differ between the $\mathrm{HC}$ subjects and the PTB patients. The $I L-4$ response was only detectable in a few subjects. In the $\mathrm{HC}$ subjects, the $I L-4$ levels did not differ among the variant genotypes of the $I L-4$ VNTR polymorphism. However, among the PTB patients, there was a trend towards an increased $I L-4$ response in patients with the B2/B1 genotype compared with the B2/B2 genotype under stimulated and unstimulated conditions (CFA, $p=0.066$; Table 2, Figure 1).

\section{Associations between the Th17 cytokine polymorphism and TB susceptibility}

For the IL6 polymorphism at nucleotide -174 , the CC genotype was significantly lower $(3.3 \%$ vs $11.0 \%$, respectively; $\mathrm{OR}=0.36 ; 95 \% \mathrm{CI}: 0.144-0.793)$ compared to $\mathrm{HC}$ group and the $\mathrm{G}$ allele was overrepresented in the LTBI group. $I L-6$ levels did not differ significantly between the genotypes of the $I L-6-174 \mathrm{G} / \mathrm{C}$ SNP in the HCs and patients.

Multilocus genotype association with the risk of LTBI and development of TB

We also assessed whether genotype combinations (in $I F N-\gamma, T N F-\alpha, I L-10$, and $I L-2)$ for the mutations in these 

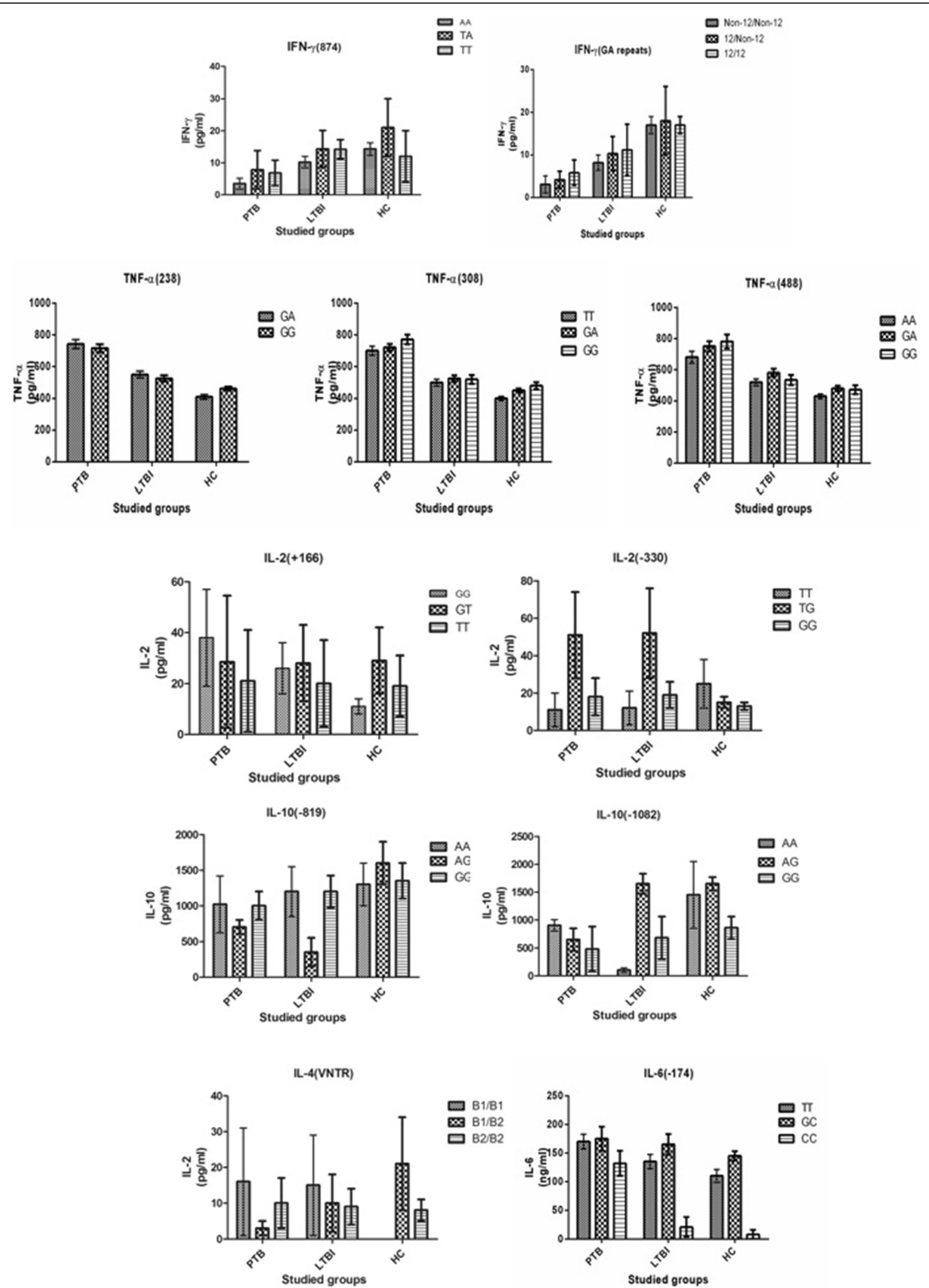

Figure 1 Influence of cytokine polymorphism on culture filtrate (CFA) of M.tuberculosis-induced cytokine production in healthy controls, LTBI and PTB patients. 
Table 3 Association between combinations of cytokine gene polymorphisms and susceptibility to LTBI and PTB

\begin{tabular}{|c|c|c|c|c|c|c|c|c|c|}
\hline & INF- $\gamma$ & TNF-a & IL-10 & IL-2 & $\mathrm{HC}$ & LTBI & PTB & LTBI.vs.Health & PTB.vs.LTBI \\
\hline & 874 & -308 & -1082 & -330 & & & & OR(95\%Cl) & OR(95\%Cl) \\
\hline 1 & $A$ & $A$ & $A$ & $G$ & 56 & 30 & 12 & $1.07(0.657-1.734)$ & $0.79(0361-1.624)$ \\
\hline 2 & A & A & A & $\mathrm{T}$ & 113 & 47 & 43 & $0.81(0.555-1.178)$ & $2.01(1.252-3.219)^{*}$ \\
\hline 3 & $A$ & $A$ & G & $\mathrm{T}$ & 50 & 41 & 5 & $1.70(1.079-2.665)^{*}$ & $0.23(0.069-0.588)$ \\
\hline 4 & $A$ & G & A & G & 70 & 32 & 22 & $9.25(5.300-15.932)^{*}$ & $1.41(1.062-2.575)^{*}$ \\
\hline 5 & A & G & A & $\mathrm{T}$ & 105 & 67 & 46 & $1.32(0.936-1.855)$ & $1.46(0.994-2.248)$ \\
\hline 6 & A & G & G & G & 29 & 28 & 7 & $1.98(1.125-3.505)^{*}$ & $0.48(0.176-1.159)$ \\
\hline 7 & A & G & G & $\mathrm{T}$ & 44 & 26 & 11 & $1.19(0.695-2.009)$ & $0.84(0.367-1.795)$ \\
\hline 8 & $\mathrm{~T}$ & $A$ & A & G & 29 & 20 & 9 & $2.47(1.300-4.599)^{*}$ & $0.89(0.353-2.101)$ \\
\hline 9 & T & A & A & $\mathrm{T}$ & 64 & 32 & 11 & $1.00(0.623-1.578)$ & $0.67(0.300-1.400)$ \\
\hline 10 & T & A & G & G & 35 & 11 & 7 & $0.62(0.281-1.263)$ & $0.33(0.107-1.945)$ \\
\hline 11 & T & A & G & $\mathrm{T}$ & 71 & 30 & 8 & $0.83(0.518-1.319)$ & $0.52(0.202-1.179)$ \\
\hline 12 & T & G & $A$ & G & 24 & 19 & 9 & $1.61(0.823-3.093)$ & $0.95(0.371-2.236)$ \\
\hline 13 & $\mathrm{~T}$ & G & A & $\mathrm{T}$ & 85 & 43 & 17 & $1.01(0.673-1.508)$ & $0.77(0.405-1.425)$ \\
\hline 14 & T & G & G & G & 38 & 20 & 6 & $1.05(0.574-1.884)$ & $0.59(0.191-1.550)$ \\
\hline 15 & T & G & G & $\mathrm{T}$ & 124 & 28 & 16 & $0.42(0.263-0.645)$ & $1.15(0.570-2.259)$ \\
\hline
\end{tabular}

Notes: HC, healthy controls; LTBI, subjects with latent tuberculosis infection; PTB, patients with pulmonary tuberculosis; INF- $\gamma$, interferon $\gamma$; TNF-a, tumor necrosis factor $a$; IL-10, interleukin 10; IL-2, interleukin 2. ${ }^{*} p<0.05$.

cytokines contribute to an increased risk of TB or LTBI. There were 15 possible combinations. There was a clear association between IFN- $\gamma-874 \mathrm{~A}$ and latent TB, especially in the combinations AAGT $(\mathrm{OR}=1.70 ; 95 \% \mathrm{CI}$ : 1.079-2.665), AGAG (OR =9.25; 95\% CI: 5.300-15.932), and AGGG (OR = 1.98; 95\% CI: 1.125-3.505), whereas $I L-10-1082$ was associated with an increased risk of PTB in the combination of AAAT $(\mathrm{OR}=2.01 ; 95 \% \mathrm{CI}$ : 1.252-3.219) and AGAG (OR = 1.41; 95\% CI: $1.062-2.575$; (Table 3).

Dose effects of genes and gene-environment interactions In addition to the multiplicative and additive models, we also analyzed the dose effects of the genotypes using the dominant and recessive models for each of the four genotypes and their interactions with BCG (Table 4). The effect of the IFN- $\gamma+874$ T allele was recessive for LTBI, whereas IFN- $\gamma+874 \mathrm{~T}$ was dominant for PTB. The $T N F-\alpha$ and $I L-2$ polymorphisms were not significant in these models (Table 4). BCG vaccination was found to compensate for the positive effect of the IFN- $\gamma$ polymorphism and was associated with a reduced risk of LTBI in the additive model.

\section{Discussion}

SNPs in several candidate genes have been linked to an increased risk of TB [12]. In this polymorphism association study, we investigated the significance of the relationship between several cytokine gene polymorphisms, their plasma products, and susceptibility to TB in a Chinese population.

The IFN- $\gamma$ polymorphism is the best-studied polymorphism in terms of its association with TB disease sites and severity. However, previous reports have been contradictory insofar as the $-874 \mathrm{~A}$ allele was more common in patients with $\mathrm{TB}$ and the T allele was more common in the controls in Italian [13], South African [14], and Spanish populations [15], whereas in China, one study reported no association [16] while a later study of the same population showed an association between TB and the A allele [17]. Our results are consistent with the latter study. In the present report, we show that the A allele at IFN- $\gamma-874 \mathrm{~A} / \mathrm{T}$ was significantly overrepresented in the populations of LTBI and PTB patients, with A allele frequencies 1.30-fold and 1.76 fold higher, respectively, than those in the HC. Significantly reduced IFN- $\gamma$ production by stimulated PBMCs was observed in individuals with the A allele at IFN- $\gamma-874$. Similar genotype distributions and associations were observed for the t875 CA repeats (non-12/12) as at $-874 \mathrm{~A} / \mathrm{T}$ in our study, and the non-12 CA repeats allele was absolutely linked to the $-874 \mathrm{~A}$ allele in our population, as previously reported in a UK population. Because the -874 A allele corresponds to lower IFN- $\gamma$ expression, the lower IFN- $\gamma$ levels may impair the activation of macrophages, resulting in the development of TB. These observations indicate that the A allele at IFN- $\gamma-874$, or another nearby genetic variation linked to it, increases the host's susceptibility to TB. 
Table 4 Dose effects of gene-gene and gene-environment interactions on susceptibility to LTBI and PTB

\begin{tabular}{|c|c|c|c|c|c|c|}
\hline \multirow[t]{2}{*}{ Cytokines } & \multirow[t]{2}{*}{ SNP position } & \multirow[t]{2}{*}{ Genotypes } & \multicolumn{2}{|c|}{ LTBI.vs.HC } & \multicolumn{2}{|c|}{ PTB.vs.LTBI } \\
\hline & & & Gene & Gene*BCG & Gene & Gene*BCG \\
\hline \multirow[t]{4}{*}{ IFNY } & $(+874) \mathrm{T}->\mathrm{A}$ & Additive & 0.092 & 0.093 & 0.264 & 0.093 \\
\hline & & Dominant & 0.616 & 0.193 & $0.010^{*}$ & 0.117 \\
\hline & & Recessive & $0.003^{*}$ & 0.277 & 0.552 & 0.405 \\
\hline & & Multiplicative & 0.174 & 0.382 & 0.976 & 0.979 \\
\hline \multirow[t]{4}{*}{ IL-10 } & $(-1082) G->A$ & Additive & 0.015 & 0.909 & 0.540 & 0.466 \\
\hline & & Dominant & 0.485 & 0.837 & 0.208 & 0.507 \\
\hline & & Recessive & 0.880 & 0.775 & 0.098 & 0.709 \\
\hline & & Multiplicative & 0.423 & 0.749 & 0.134 & 0.411 \\
\hline \multirow[t]{4}{*}{ TNFa } & $(-308) A->G$ & Additive & 0.845 & 0.722 & 0.945 & 0.873 \\
\hline & & Dominant & 0.995 & 1.000 & 0.995 & 0.999 \\
\hline & & Recessive & 0.072 & 0.326 & 0.144 & 0.700 \\
\hline & & Multiplicative & 1.000 & 0.999 & 0.995 & 0.994 \\
\hline \multirow[t]{4}{*}{ IL2 } & & Additive & 0.815 & 0.909 & 0.437 & 0.485 \\
\hline & & Dominant & 0.485 & 0.837 & 0.845 & 0.613 \\
\hline & & Recessive & 0.881 & 0.775 & 0.253 & 0.223 \\
\hline & & Multiplicative & 0.423 & 0.749 & 0.577 & 0.397 \\
\hline
\end{tabular}

Notes: HC, healthy controls; LTBI, subjects with latent tuberculosis infection; PTB, patients with pulmonary tuberculosis; INF- $\gamma$, interferon $\gamma$; IL-10, interleukin 10; TNF-a, tumor necrosis factor $a ;$ IL-2, interleukin 2. ${ }^{*} p<0.05$.

Because the modulation of the T-cell response by $I L-10$ seems to influence the susceptibility of the host to TB infection [18], identifying the polymorphisms in the $I L-10$ gene may be useful in predicting disease susceptibility. The frequency of the $I L-10-1082 \mathrm{G}$ allele in our population (4\%) was similar to that in Korean (7.4\%) [19] and Japanese (6.5\%) populations [20], but was significantly different from that reported in Caucasians (48\%) [21]. The present results also show that the genotype frequency of the $I L-10-1082 \mathrm{~A}$ allele was significantly increased in TB patients compared with the LTBI subjects, especially when it was associated with the IFN- $\gamma+874 \mathrm{~A}$ allele $(\mathrm{OR}=3.59$; $p=0.045)$. This is consistent with the low levels of mycobacterial-stimulated IFN- $\gamma$ and $I L-10$ in PTB patients. In fact, there remains a discrepancy with the -1082 polymorphism in the IL10 promoter, for which no association with TB was reported in Gambian [22] and Korean populations [23]. However, an association between the $I L-$ $10-1082 \mathrm{~A}$ allele and TB was observed in an Italian (Sicilian) [24] population, and GA heterozygosity was associated with PTB in Cambodia [25]. These observations may reflect ethnic-specific genetic variations or suggest that other more-distal promoter elements are involved.

A number of studies have investigated the association between the TNF- $\alpha 308$ (rs1800629 G/A) polymorphism and susceptibility to TB in different populations. However, the association between TB with TNF- $\alpha$ differs with ethnicity, and many of these studies have produced inconsistent results [26]. In the present study, a significant negative association between TB and the TNF- $\alpha 308$ GA genotype and a significant positive association with the GG genotype were found, and the $\mathrm{G}$ allele was carried by $90 \%$ of patients and by $85.8 \%$ of controls. The A allele is associated with high TNF- $\alpha$ production and the G allele is associated with low TNF- $\alpha$ production. The high frequency of the genotype associated with increased protein production may be attributable to selection during the course of evolution, increasing the frequency of the most advantageous genotype. Our data indicate that TNF- $\alpha$ production was reduced in our PTB patients. Although the study by Scola et al. demonstrated a reduction in the low-producing - 308 G/G TNF- $\alpha$ homozygous individuals in the affected group [24], and the study of Bikmaeva et al. revealed an association between the high-producing A allele and a higher risk of PTB [27], although several studies have failed to confirm the association between TNF- $\alpha$ and TB [28].

In the present study, we also analyzed several other candidate gene polymorphisms and their association with the susceptibility to PTB or LTBI. We found a significant negative association between the $-330 \mathrm{TT}$ genotype of $I L-2$ and PTB; the T allele was significantly overrepresented in the LTBI group and the HC relative to the PTB group. This suggests that the GG haplotype is associated with a susceptibility to PTB. Although translation studies have demonstrated the increased activity of the $\mathrm{G}$ allele, allele expression studies have shown higher expression of $I L-2$ in the PBMCs of individuals 
with the $I L-2-330$ TT genotype than in those of individuals with the GG genotypes, suggesting that the $I L-2$ promoter activity is also influenced by other polymorphisms, probably $+160 \mathrm{~T} / \mathrm{G}$ [29]. No significant association between TB and IL-4 levels was observed for the VNTR polymorphism. The results of the present study do not preclude the possible influence of the VNTR polymorphism on the $I L-4$ response because $I L-4$ was undetectable in most of the subjects tested, which could be attributable to the downregulation of the Th2 responses by the IFN- $\gamma$ secreted in response to the antigens used. In our study, the $I L-6-174$ CC genotype was significantly negatively associated with the LTBI group and with the reduced production of $I L-6$. In previous studies, significant levels of $I L-6$ were shown to be produced in response to Mtb infection. Human and murine macrophages secrete $I L-6$ in response to Mtb in vitro [30], and elevated concentrations of $I L-6$ are present in the plasma of patients with TB [31]. It has also been reported that the induction of $I L-6$ activity by Mtb inhibits IFN- $\gamma$ signaling, so the bacterium evades eradication by the cellular immune response [32]. We have reported that the IL-12 AA genotype is associated with LTBI susceptibility. This is consistent with reports from Japan and France, where polymorphisms in the $I L-12$ receptor $\beta 1$ gene (IL-12RB1) are known to influence the susceptibility to and severity of TB [33].

A consensus seems to be emerging that the combined effects of several cytokine SNPs may play a crucial role in susceptibility to LTBI and TB diseases [29]. The combination of the IFN- $\gamma$ polymorphism with other cytokine polymorphisms was most effective in predicting LTBI. This observation is consistent with the recently described functions of IFN- $\gamma$ in a mouse model, in which mice with a disrupted $I F N-\gamma$ gene were unable to control Mtb infection [34]. The combination of IL-10 polymorphisms with other cytokine polymorphisms may predict a susceptibility to TB. These results also substantiate an earlier report [35] that implicated $I L-10$ in the reactivation of TB in humans and in TB disease in a mouse model. A recently published meta-analyses reinforced the critical importance of $I F N-\gamma+874$ T/A as a genetic marker of TB resistance [8], and stressed that although $I L-10$ has a specific effect on the form and severity TB, it does not affect susceptibility per se. Our results are consistent with these findings. BCG vaccination cannot be ignored [36]. However, we found no evidence that BCG vaccination interacts with gene polymorphisms in affecting susceptibility to TB.

In this study, all cases of TB disease were included, but the majority of patients had PTB. Therefore, it was not possible to demonstrate an association between the system involved and cytokine polymorphisms. A larger series is required to test the possible associations between cytokine gene polymorphisms and organ preference in TB, and the clinical severity of the disease.

\section{Conclusions}

Single-nucleotide functional polymorphisms in cytokine genes display variable associations with LTBI and active TB disease. Combinations of cytokine SNPs with the IFN- $\gamma+874$ T/A SNP markedly influence the severity and outcome of TB. Our results may reflect the polygenic aspects of a predisposition to severe and active TB.

\section{Methods}

\section{Study subjects}

Two case-control studies consisted of 120 patients diagnosed with PTB (patient group), 240 with LTBI (the control group for the patient group, and the case group for the $\mathrm{HC}$ group), and $480 \mathrm{HC}$ individuals (control group). The subjects were recruited during the routine investigation of individuals in contact with TB in seven districts of Shanghai between 2009 and 2010. The subjects in the patient group were bacteriologically confirmed as TB patients infected with Mtb. None of the patients reported infection with human immunodeficiency virus. LTBI was identified in the contacts of TB patients, and had no TB-related symptoms but a positive T-SPOT.TB test. The subjects in the $\mathrm{HC}$ group were also recruited from the contacts of $\mathrm{TB}$ patients, with no TB-related symptoms or previous history of TB, and a negative T-SPOT.TB test.

\section{T-SPOT.TB assays}

T-SPOT.TB assays were performed according to the manufacturer's instructions (Oxford Immunotec, Oxford, UK). Briefly, PBMCs were isolated from whole blood by centrifugation with Lymphocyte Separation Medium (Ficoll 400), washed twice, and incubated with ESAT-6 and CFP-10 antigens for $16-20 \mathrm{~h}\left(37^{\circ} \mathrm{C}, \mathrm{CO}_{2}\right)$ in an enzyme-linked immunosorbent spot assay. The spots were counted. The T-SPOT.TB result was positive when the number of spots in the test well (against at least one of the two tested antigens) was at least six, if the negative control had fewer than five spots, or twice the number of spots in the negative control wells, if the negative control had more than six spots. This cutoff was predefined in the manufacturer's instructions.

\section{Whole blood stimulation assays}

The stimulated whole blood (WB) culture assay used to assess the cytokine profiles has been described in detail previously [37]. Briefly, heparinized blood was diluted 1:11 with sterile RPMI 1640 tissue culture medium containing 100 units $/ \mathrm{ml}$ penicillin $/ 100 \mathrm{mg} / \mathrm{ml}$ streptomycin and $2 \mathrm{mM}$ L-glutamine (Sigma Chemical Co., St. Louis, MO, USA). Within $2 \mathrm{~h}$ of collection, diluted WB 
(900 ml/well) was stimulated with Mtb culture filtrate proteins $(5 \mathrm{mg} / \mathrm{ml})$ in a 24-well tissue culture plate. The supernatants were collected from the wells at varying intervals.

\section{Cytokine assessment}

The cytokines in the supernatants were assessed using pairs of monoclonal antibodies, as described in detail previously [38]. Dose-response curves were constructed for each individual plate. The supernatants were serially diluted and the optical density (OD) readings in the linear ranges of the dose-response curves were used to calculate the concentrations. The final concentrations $(\mathrm{pg} / \mathrm{ml})$ were obtained by multiplying the values by the dilutions at which the ODs were read. The sensitivity range of cytokine detection was $7.5-1000 \mathrm{pg} / \mathrm{ml}$ and was similar to that reported by the manufacturer.

\section{Molecular methods}

Genomic DNA was extracted from blood samples with the Puregene Genomic DNA Isolation Kit (Gentra Systems, Minneapolis, MN, USA), according to the manufacturer's instructions. Primers were purchased from MWG-Biotech AG (Ebersberg, Germany). IFN- $\gamma$ (rs2430561), IL-10 (rs1800896), and TNF (rs1800629) were genotyped with amplification-refractory mutation system-polymerase chain reaction (ARMS-PCR), with human growth hormone or bactin primers as the internal controls to check the accuracy of the PCR reactions. IL-6 (rs1800795) was genotyped with tetraARMS-PCR. The amplified products (5 ul, undiluted) were monitored with electrophoresis on agarose gel prepared in Tris-acetate ethylene-diaminetetraacetic acid (TAE) buffer containing $10 \mathrm{mg} / \mathrm{ml}$ ethidium bromide. The product bands were visualized on a UV transilluminator and photographs were taken for genotype interpretation.

\section{Confirmatory sequencing methodology}

DNA sequencing was applied to a subset of samples (10\%-15\% for each SNP) to confirm the genotypes identified with ARMS and tetra-ARMS-PCR. The primers used for the sequencing reactions for $I F N-\gamma$ and $I L-10$ were designed using the Lasergene version 7.0 software (DNASTAR, Madison, WI, USA), and the primers for TNF and $I L-6$ were designed using the web-based BatchPrimer3 software. Sequence data produced with the ABI 3130xl Genetic Analyzer were reviewed for confidence levels with the ABI Sequence Scanner software, and the sequencing results were analyzed with pairwise alignments of the sequences with ClustalW version 1.83. The allelic specificities of the cytokine gene SNPs were determined by comparison of the PCR results with the nucleotide sequencing results for the alleles. Concordance of $>95 \%$ was observed in all cases.

\section{Statistical analysis}

Data were analyzed with SPSS for Windows version 16 (SPSS Inc., Chicago, IL, USA). The cytokine levels were expressed as means \pm standard error. Hardy-Weinberg proportions were determined by applying the equation $\left(\mathrm{p}^{2}+2 \mathrm{pq}+\mathrm{q}^{2}\right)$. All polymorphisms were tested for Hardy-Weinberg equilibrium separately in the patient and control groups by comparing observed and expected numbers using the $x^{2}$ test. The distributions of the cytokine gene polymorphisms were compared between the different study groups with the $x^{2}$ test or Fisher's exact test if a cell was below 5 . Values of $p$ were smaller than 0.05 were considered significant. Odds ratios (OR) and 95\% confidence intervals (CI) were also calculated. A logistic regression analysis was used to determine the effects of age and sex on the genotypes. Combinations of the IFN- $\gamma+874$ TA genotype with the IL10 21082 AG, TNF -308 GA, or IL-6 2174 GC genotype were used to determine the two-gene combination effects. Nine possible genotypes were compared between the controls and PTB patients using Pearson's $X^{2}$ or Fisher's exact test.

\section{Ethics committee approvals}

The study protocol was approved by the Ethics Committee of the School of Public Health, Fudan University, and written informed consent was obtained from all study participants.

\section{Abbreviations \\ TB: Tuberculosis; Mtb: Mycobacterium tuberculosis; IFN-ү: Interferon-gamma; IL: Interleukin; LTBI: Latent tuberculosis infection patients; PTB: Pulmonary tuberculosis; HC: Healthy control; BCG: Bacille Calmette- Guérin.}

\section{Competing interests}

The authors declare that they have no competing interests.

\section{Authors' contributions}

$\mathrm{YH}, \mathrm{LW}$, and $\mathrm{BX}$ designed the study, conceived experiments and prepared the manuscript. YH and LW carried out genotyping, sequencing and data analysis. YH, DL, QZ and WJ extracted DNA from blood samples and completed the whole blood stimulation assays, TSPOT.TB assays and performed data analysis. YH and LW drafted the manuscript. $\mathrm{DL}$ and $\mathrm{ZQ}$ also contributed to manuscript writing. All authors read and approved the final manuscript.

\section{Acknowledgements}

We gratefully acknowledge the participation of all subjects. Thanks to the health staff in municipal and district CDCs for collecting data and blood samples. This study was supported by the China National Key Project for Infectious Disease (grant No. 2013ZX10004903), Shanghai Leading Academic Discipline Project (No. B118), young investigator grant from Health and Family Planning Commission of Shanghai (20114Y034) and the National Natural Science Foundation of China (grant No. 81373063).

Received: 9 August 2014 Accepted: 30 January 2015

Published online: 22 February 2015 


\section{References}

1. Zumla A, George A, Sharma V, Herbert N, Baroness Masham of I. WHO's 2013 global report on tuberculosis: successes, threats, and opportunities. Lancet. 2013;382(9907):1765-7.

2. Bellamy R. Genetic susceptibility to tuberculosis. Clin Chest Med. 2005;26 (2):233-46. vi.

3. Wilkinson RJ. Human genetic susceptibility to tuberculosis: time for a bottom-up approach? J Infect Dis. 2012;205(4):525-7.

4. Sher A, Coffman RL. Regulation of immunity to parasites by $T$ cells and $T$ cell-derived cytokines. Annu Rev Immunol. 1992;10:385-409.

5. Cooper AM, Khader SA. The role of cytokines in the initiation, expansion, and control of cellular immunity to tuberculosis. Immunol Rev. 2008;226:191-204.

6. Doffinger R, Helbert MR, Barcenas-Morales G, Yang K, Dupuis S, Ceron-Gutierrez $L$, et al. Autoantibodies to interferon-gamma in a patient with selective susceptibility to mycobacterial infection and organ-specific autoimmunity. Clin Infect Dis. 2004;38(1):e10-4

7. Karimi MH, Hejr S, Geramizadeh B, Yaghobi R, Sagheb MM, KamaliSarvestani E. Combined analysis of cytokine gene polymorphism and the level of expression with allograft function in kidney transplant recipients. Transpl Immunol. 2014;30(1):46-51.

8. Pacheco AG, Cardoso CC, Moraes MO. IFNG +874 T/A, IL10-1082G/A and TNF -308G/A polymorphisms in association with tuberculosis susceptibility: a meta-analysis study. Hum Genet. 2008;123(5):477-84.

9. Matos Gl, Covas Cde J, Bittar Rde C, Gomes-Silva A, Marques F, Maniero VC, et al. IFNG +874 T/A polymorphism is not associated with American tegumentary leishmaniasis susceptibility but can influence Leishmania induced IFN-gamma production. BMC Infect Dis. 2007;7:33.

10. Kumar V, Khosla R, Gupta $\vee$, Sarin BC, Sehajpal PK. Differential association of tumour necrosis factor-alpha single nucleotide polymorphism (-308) with tuberculosis and bronchial asthma. Natl Med J India. 2008;21(3):120-2.

11. Moller M, Nebel A, van Helden PD, Schreiber S, Hoal EG. Analysis of eight genes modulating interferon gamma and human genetic susceptibility to tuberculosis: a case-control association study. BMC Infect Dis. 2010;10:154.

12. Hill AV. The immunogenetics of human infectious diseases. Annu Rev Immunol. 1998:16:593-617.

13. Lio D, Marino V, Serauto A, Gioia V, Scola L, Crivello A, et al. Genotype frequencies of the $+874 \mathrm{~T}-->$ A single nucleotide polymorphism in the first intron of the interferon-gamma gene in a sample of Sicilian patients affected by tuberculosis. Eur J Immunogenet. 2002;29(5):371-4.

14. Rossouw M, Nel HJ, Cooke GS, van Helden PD, Hoal EG. Association between tuberculosis and a polymorphic NFkappaB binding site in the interferon gamma gene. Lancet. 2003;361(9372):1871-2.

15. Lopez-Maderuelo D, Arnalich F, Serantes R, Gonzalez A, Codoceo R, Madero $\mathrm{R}$, et al. Interferon-gamma and interleukin-10 gene polymorphisms in pulmonary tuberculosis. Am J Respir Crit Care Med. 2003;167(7):970-5.

16. Wu F, Qu Y, Tang Y, Cao D, Sun P, Xia Z. Lack of association between cytokine gene polymorphisms and silicosis and pulmonary tuberculosis in Chinese iron miners. J Occup Health. 2008;50(6):445-54.

17. Yang Y, Li X, Cui W, Guan L, Shen F, Xu J, et al. Potential association of pulmonary tuberculosis with genetic polymorphisms of toll-like receptor 9 and interferon-gamma in a Chinese population. BMC Infect Dis. 2013;13:511.

18. Fortsch D, Rollinghoff M, Stenger S. IL-10 converts human dendritic cells into macrophage-like cells with increased antibacterial activity against virulent Mycobacterium tuberculosis. J Immunol. 2000;165(2):978-87.

19. Pyo CW, Hur SS, Kim YK, Choi HB, Hong YS, Kim DW, et al. Polymorphisms of IL-1B, IL-1RN, IL-2, IL-4, IL-6, IL-10, and IFN-gamma genes in the Korean population. Hum Immunol. 2003;64(10):979-89.

20. Ide A, Kawasaki E, Abiru N, Sun F, Takahashi R, Kuwahara H, et al. Genetic association between interleukin-10 gene promoter region polymorphisms and type 1 diabetes age-at-onset. Hum Immunol. 2002;63(8):690-5.

21. Chong WP, Ip WK, Wong WH, Lau CS, Chan TM, Lau YL. Association of interleukin-10 promoter polymorphisms with systemic lupus erythematosus. Genes Immun. 2004;5(6):484-92

22. Bellamy R. Susceptibility to mycobacterial infections: the importance of host genetics. Genes Immun. 2003;4(1):4-11.

23. Shin HD, Park BL, Kim YH, Cheong HS, Lee IH, Park SK. Common interleukin 10 polymorphism associated with decreased risk of tuberculosis. Exp Mol Med. 2005:37(2):128-32.
24. Scola L, Crivello A, Marino V, Gioia V, Serauto A, Candore G, et al. IL-10 and TNF-alpha polymorphisms in a sample of Sicilian patients affected by tuberculosis: implication for ageing and life span expectancy. Mech Ageing Dev. 2003:124(4):569-72

25. Goldfeld AE. Genetic susceptibility to pulmonary tuberculosis in Cambodia. Tuberculosis. 2004:84(1-2):76-81.

26. Varahram M, Farnia P, Nasiri MJ, Karahrudi MA, Dizagie MK, Velayati AA. Association of Mycobacterium Tuberculosis Lineages with IFN-gamma and TNF-alpha Gene Polymorphisms among Pulmonary Tuberculosis Patient. Mediterr J Hematol Infect Dis. 2014;6(1):e2014015.

27. Bikmaeva AR, Sibiriak SV, Valiakhmetova D, Khusnutdinova EK Polymorphism of the tumor necrosis factor alpha gene in patients with infiltrative tuberculosis and from the Bashkorstan populations. Mol Biol. 2002;36(5):784-7

28. Henao MI, Montes C, Paris SC, Garcia LF. Cytokine gene polymorphisms in Colombian patients with different clinical presentations of tuberculosis. Tuberculosis. 2006;86(1):11-9.

29. Matesanz F, Fedetz M, Leyva L, Delgado C, Fernandez O, Alcina A. Effects of the multiple sclerosis associated -330 promoter polymorphism in IL2 allelic expression. J Neuroimmunol. 2004;148(1-2):212-7.

30. Giacomini E, lona E, Ferroni L, Miettinen M, Fattorini L, Orefici G, et al. Infection of human macrophages and dendritic cells with Mycobacterium tuberculosis induces a differential cytokine gene expression that modulates T cell response. J Immunol. 2001;166(12):7033-41.

31. El-Ahmady O, Mansour M, Zoeir H, Mansour O. Elevated concentrations of interleukins and leukotriene in response to Mycobacterium tuberculosis infection. Ann Clin Biochem. 1997;34(Pt 2):160-4.

32. Nagabhushanam V, Solache A, Ting LM, Escaron CJ, Zhang JY, Ernst JD. Innate inhibition of adaptive immunity: Mycobacterium tuberculosisinduced IL-6 inhibits macrophage responses to IFN-gamma. J Immunol. 2003:171(9):4750-7.

33. Kusuhara K, Yamamoto K, Okada K, Mizuno Y, Hara T. Association of IL12RB1 polymorphisms with susceptibility to and severity of tuberculosis in Japanese: a gene-based association analysis of 21 candidate genes. Int J Immunogenet. 2007:34(1):35-44.

34. Rottman M, Soudais C, Vogt G, Renia L, Emile JF, Decaluwe H, et al. IFN-gamma mediates the rejection of haematopoietic stem cells in IFN-gammaR1-deficient hosts. PLoS Med. 2008;5(1):e26.

35. Turner J, Gonzalez-Juarrero M, Ellis DL, Basaraba RJ, Kipnis A, Orme IM, et al. In vivo IL-10 production reactivates chronic pulmonary tuberculosis in C57BL/6 mice. J Immunol. 2002;169(11):6343-51.

36. Menzies D, Nahid P. Update in tuberculosis and nontuberculous mycobacterial disease 2012. Am J Respir Crit Care Med. 2013;188(8):923-7.

37. Hussain R, Kaleem A, Shahid F, Dojki M, Jamil B, Mehmood H, et al. Cytokine profiles using whole-blood assays can discriminate between tuberculosis patients and healthy endemic controls in a BCG-vaccinated population. J Immunol Methods. 2002;264(1-2):95-108.

38. Hussain R, Talat N, Shahid F, Dawood G. Longitudinal tracking of cytokines after acute exposure to tuberculosis: association of distinct cytokine patterns with protection and disease development. Clin Vaccine Immunol. 2007;14(12):1578-86.

\section{Submit your next manuscript to BioMed Central and take full advantage of:}

- Convenient online submission

- Thorough peer review

- No space constraints or color figure charges

- Immediate publication on acceptance

- Inclusion in PubMed, CAS, Scopus and Google Scholar

- Research which is freely available for redistribution 